\title{
Applied Binarology: Theoretical Aspects
}

\author{
Virginia Trimble \\ University of California, Irvine, Irvine, CA, USA \\ and \\ Las Cumbres Observatory, Goleta, CA
}

email: vtrimble@uci.edu

\begin{abstract}
The winding up of a conference like this provides the opportunity to look (1) backwards at how we reached the present stage of understanding of binary star behavior and its relationship to the rest of astronomy, (2) around at the garden of unsolved problems, and (3) cautiously forward at what might come next.
\end{abstract}

Keywords. binary stars

\section{Introduction}

Like Dr. Scarfe, the previous speaker, I attended my first binary star conference nigh on to 40 years ago. It was IAU Colloquium 6, Mass Loss and Evolution in Close Binaries, held in Elsinore, Denmark, 15-19 September 1969. The cover of the proceedings shows a classic evolutionary sequence, taken from the talk of Bohdan Paczyński, in which a primary fills its Roche lobe, dumps material onto the secondary on a thermal time scale until the mass ratio is reversed, and then continues more gentle donation on the nuclear time scale (conservatively, of course).

This is clearly a generation ago, since, those present at S240 included, of the Elsinore participants, only Alan Batten and Robert Wilson of the relatively senior people, Petr Harmanec (who, with the late Jiř́ Horn represented the Prague group and has somehow in the interim grown from a callow postdoc to a distinguished director), Johannes Andersen (then a graduate student, helping with logistics), and the present writer (another new postdoc who wasn't actually invited to Colloq. 6 but came anyway). The topic, as shown in the poster presentation by Pustylnik \& Pustynski and by de Mink \& Pols, remains, however, a focus of on-going research. Incidentally, Daniel Popper presented the class of RS CVn stars (near the main sequence, with emission lines in one or both components) there, in a table with 22 examples, so anyone who tries to tell you the class was discovered later by someone else should be referred to the proceedings (Gyldenkerne \& West 1970).

\section{Golden Moments in Theory of Binary Stars}

That most close pairs of stars (as well as triples and clusters) are physical systems was a theoretical discovery, reported by the Rev. John Michell, B.D.F.R.S. (1768) in 1767. The paper, which also obtained stellar distances from the assumption that other stars were really about as bright as the sun, considered $\beta$ Cap as a possible accidental juxtaposition of stars at different distances, and by the time the author had considered other doubles, triples, and richer groups, he reached the conclusion that the odds were many million millions to one against the chance hypothesis. The arithmetic is essentially the same as that used to show that, with 20-some people in a room, two of them will probably have the same birthday. The cause was left open, "...their mutual gravitation 
or some other law or appointment of the Creator." Well, that 'B.D.' is, after all, bachelor of divinity.

In 1782, Goodricke and Pigott recognized the periodicity of Algol and suggested eclipses by an opaque, less luminous orbiting body as the cause. They backed off from their correct hypothesis after failing to be able to account for the light curve of $\delta$ Cephei the same way, and rotating, spotted stars were the "best buy" hypotheses for periodic variables through most of the 19th century.

Peeking for a moment over the fence at the observers, we find Christian Mayer publishing his first double star catalog in 1778 and remarking that some of the close pairs seemed to have experienced relative motion since the time of Flamsteed. Credit for recognizing actual orbital motion is customarily assigned to William Herschel in an 1803 paper. Several of these items, incidentally, come from the incipient Biographical Encyclopedia of Astronomers (Hockey et al. 2007).

Pickering revived the hypothesis of eclipsing stars for periodic variability in the 1880s, but by 1914, Shapley had shown that the Cepheids, for instance, would have to have a sin i less than the stellar radii and $\mathrm{f}(M) \leqslant 0.001$. He proposed pulsation as the solution and we now, of course, recognize that star spots, eclipses, and pulsation can all yield periodic light curves. The lesson of "all of the above" should perhaps be carried forward to other phenomena to be mentioned later.

Stars try to expand as they age, and Kuiper in 1941 appears to have been first to point out that this would get them into trouble with the interior Lagrangian surfaces (Roche lobes) if two stars orbited with semi-major axis less than the desired final radius. Crawford in 1955 applied this idea to resolve the then-worrisome Algol paradox (the less massive star the more evolved), and the first detailed calculations came from Morton in 1960, neither of them names primarily associated with binary star evolution (but see Paczyński 1971 if you doubt). And in 1964 Fred Hoyle, enthusiastic as ever, proposed a dog-eat-dog series of processes, in which material spilled back and forth between the stars many times.

The field took off in 1966, with independent but similar computations of binary evolution in three places, Munich under Kippenhahn, Ondřejov under Plavec, and Warsaw under Paczyński. Whether the primary filled its Roche lobe before hydrogen core exhaustion, while a red giant, or still later in life made major differences to the outcomes. And it was right here in Prague at the 1967 General Assembly that Anne Underhill most forcefully disagreed that Wolf-Rayet stars might be one of those outcomes, saying that "there are more models that aren't stars than there are stars that aren't models." She lost the WR battle, but the general point is worth remembering!

Some early computations in which mass and angular momentum were allowed to leave the system seemed to show no qualitative differences. This changed in the mid 1970s, and Paczyński, speaking at the first all-European meeting on astronomy, in Leicester in 1975, pointed to systems like V471 Tauri that simply could not have reached their current values of mass and size without very significant removal of angular momentum as well as mass. This was the meeting during which the transient X-ray source, A0620-00 rose to be the brightest thing in the sky in the Ariel-5 (partly a Leicester project) energy band. And the name common envelope binary quickly came to describe the - very short lived - process during which most of the losses occur.

On the assumption that the next truly golden moment has been the present symposium, we now fast-forward to significant issues that seemed ripe for discussion here and now. 


\section{Further Current Concerns}

This is the list I arrived with of items about which something is known but more is desired, only slightly re-ordered. It is given with NASA bullets because, while the thoughts are in some sense complete, the sentences are not.

- Calibration of stellar structure and evolution calculations from observations of $L, T$, and $R$ vs. mass, composition, and age (where information is available); the core reason why our colleagues tolerate binary star astronomers!

- Input for computations of chemical evolution and stellar population synthesis, from which binaries tend to be systematically excluded, sometimes leading to wrong answers (e.g., age if you leave out the blue stragglers).

- Tests of star formation theory from percentage of binaries vs. primary mass and the distribution of separations, mass ratios, and eccentricities.

- Clarification of scenarios leading to cataclysmic variables, type Ia supernovae (important for cosmology as well as nucleosynthesis), some gamma ray bursts, some sdOBs, and some blue stragglers.

- Disentangling the effects of rotation from age per se on stellar activity.

- Discovery of stellar-mass black holes, Cyg X-1 in 1972, two of the discoverers, C.T. Bolton and Paul Murdin having come to Prague in 1967, but not to S240.

- Determination of the equation of state of dense nuclear matter from the maximum mass and $\mathrm{M} / \mathrm{R}$ ratios of neutron stars in X-ray binaries.

- Discovery of other relativistic effects, including dragging of inertial frames in BHXRBs and gravitational radiation from double neutron-star binary pulsars, although we ought to remember that the effect on the orbits of cataclysmic variables was recognized first, by a decade or more (depending on whom you credit with the recognizing).

- The radial distribution of density, with implications for rotation, convection, and mixing from apsidal motion.

- Limb darkening, convection mapping, spotting, and other deviations from uniformly bright spherical surfaces from tomography and the Rossiter-McLaughlin effect. (look it up!)

- Response of the secondary to receiving material on a time scale shorter than its own thermal one at the onset of rapid mass transfer (apparently not quite so devastating these days as Benson 1970 found).

- Continuity, or absence thereof, of brown dwarfs with low-mass M dwarfs, based on percentages of binaries and distributions of separations, etc.

- Calibration of distance scales with clean (detached) double line SBs that also eclipse.

- Probes of the current and past gravitational potential of the Milky Way from survival of wide binaries etc.

\section{Recent progress and Future Prospects}

These get paragraphs rather than bullets, because many of them have verbs. Many were also précised in Guinan's introductory talk. And the ordering would be difficult to defend. The posters were identified by abstract numbers during my talk. These have been replaced by surnames of the first author, on the grounds that neither of the readers is likely to have the Prague abstract book, which, being bound in signatures, defied disassembly and partial return, requiring participants to adopt an "all or nothing" approach to bringing it back.

The most striking single number in my view was the extreme deficiency of early-type eclipsing binaries in the LMC (Mazeh, using an OGLE sample). This is, admittedly, an 
observation, but it prompts several quasi-theoretical questions besides "What did he do wrong?" (OGLE cannot have missed 10 times as many EBs as it reported, but could the parent population somehow have been greatly overestimated?) More to the point, the LMC is known to have lots of supersoft X-ray binaries, which are advertised as coming from not too much further down the main sequence. Can these numbers be reconciled?

Formation ought to come first. Very little was said about binary formation at S237 (star formation in general), but we had a fine talk from Clarke, who emphasized the need for more and better statistics of binary populations in various contexts. Well, I tried long ago, but gave it up when a distinguished colleague (now deceased) began a review by referring to "the incorrect methods of Trimble." We made it up in the cafeteria line at the Patras GA, but belated thanks, Helmut, for fielding that fly ball long ago. The posters by Köhler et al. and Kouwenhoven et al. provided some of the requested statistics for Orion and Sco 0B2. A real surprise was the seemingly flat distribution function of angular momenta (Zwitter).

Next one might put the effects on formation and dynamical evolution of planets. Definitely non-negligible according to posters by Neuhaeuser et al. and Fabrycky. And the ratio of "big" to "little" semi-major axis in a hierarchical triple remains out there somewhere around the 7:1 that I learned from R.S. Harrington long ago - 10:1 according to Lane, and one of the implications is that some systems must have arisen by star exchange, because, although the system is stable now, the planet could not have formed there.

Since core uses include calibration of stellar structure and evolution calculations and, more recently, of the extra-galactic distance scale, it is essential to know just how accurate masses (etc.) from eclipsing SB2's can be made and whether most star pairs can be fitted with somebody's isochrones with a consistent age and composition for the two stars. It is, I think, tempting to focus on moderate discrepancies (posters by de Mink, Kholtygin, Lacy, Richichi, Kiyaeva, and Lee and co-authors). My own prejudice is that we should trumpet this area as one of the major triumphs of modern astrophysics: to a very considerable extent, the stars we calculate are the stars we see, although, of course, the calculating and the seeing can always be done better.

The final white dwarf mass produced by a given main sequence star mass is not as firmly established as you might suppose (Catalan), partly because the real range of WD masses is much narrower than one might have expected.

Duplicity can interact with stellar pulsation ("asteroseismology") by driving particular resonant frequencies (a surprise to me) and in other ways (talk by Aerts, posters by Ulas, Pigulski, and Latkovic). The interaction between duplicity and stellar activity goes both ways. Oláh reported preferred longitudes for spots correlated with binary phase; and Kafka showed that mass transfer in CVs can nearly turn off when there is a large spot under L1.

The role of binaries in chemical evolution of galaxies and population synthesis, and its wide neglected by evolvers and synthesizers, was superbly reviewed by Vanbeveren. I don't know what to do about it except to holler periodically, "Don't forget the binaries!" The posters by Han et al. and Eggleton et al. addressed related issues, although we all heard, at the end of oral presentation of some of the latter work the announcement by R.F. Griffin that the first mistake in the table occurred in line three (a companion that has not been confirmed).

Within the binary systems themselves, there is a rapid mass transfer phase, of which $\beta$ Lyrae remains the classic example (Chadima et al. poster). It is followed by a slow mass transfer phase and, perhaps sometimes, by back transfer to the primary of material flowing off the secondary or a disk (Qian et al. poster). And somewhere in the poster 
forest was a previously-advertised $\beta$ Lyrae star that is probably just a contact system (as if we understood those properly).

Dynamical issues within systems include circularization, synchronization, and apsidal motion (posters by Roman, Chatterjee, and Farbiash). Some processes, especially disruption, are very sensitive to the presence of a surrounding star cluster, even a modest one (Kroupa). The role of binaries in encouraging or preventing core collapse in globular clusters belonged to JD14 on dense stellar systems, which was summarized by Douglas Heggie, a, perhaps the, pioneer of such studies.

Some astronomical phenomena happen only in binaries and, if you believe most of the published papers, each can happen only one way (that, of course, proposed by the authors). Following the Gell-Mann dictum that "everything that isn't forbidden is compulsory" (he meant particle physics processes), I suspect that multiple channels to a given result may be the norm. A few examples from the symposium included W UMa stars (Li poster); CVs (Bisikalo talk, Skopal and Unda-Sanzana posters); blue stragglers (not the focus of any presentation but one of my personal favorites to come from mass transfer; binary and triple mergers, and even perhaps binary-induced mixing); and, especially, Type Ia supernovae (Vanbeveren talk and several of the presentations at JD09), if only because each of about four major suggested progenitor classes presents problems (excess hydrogen for main sequence donors right on down to non-existence for double degenerates of large total mass and short period).

On the more cheerful side, processes in binaries can typify ones that occur across the range of astrophysics, including accretion and disks (Torok and Eze posters) and magnetized jets (Viallet and Lopez posters), as emphasized in Zhang's Invited Discourse (which, like the proceedings of the various JDs mentioned here should appear eventually in Highlights, van der Hucht 2007).

That wide binaries have survived in the Galactic halo population, given all the unpleasantness of mergers and such, is perhaps a bit of a surprise, but survived some of them have (talks by Chanamé, Bisikalo, Poveda, posters by Oswalt and Orlov, the latter actually on binary pulsars).

That binaries are, one way or another, relevant to the acceleration of run-away stars was addressed by McSwain (who was forced to conclude that very few OB runaways have carried their neutron star partners with them), and it is a special pleasure to note that the originators of both of the main hypotheses, Arcadio Poveda (cluster processes) and Adriaan Blaauw (liberation by a supernova) were in Prague, although the latter only during the first week.

Finally come tests of general relativity. I continue to find it remarkable that GR effects (for both "advance of the perihelion" and for "loss of angular momentum in gravitation radiation") should be detectable in cataclysmic variables, but Giménez left us in no doubt that it is so, and that the effects are more or less as expected. And the Holley-Bockelmann poster said that CVs will be a significant background for LISA. I think that the paper by Wana et al. (which was displayed, although not with most of the S240 ones) was advocating something other than GR. And the Křížek poster made the very important point that what is sometimes being tested is not the GR but our ability to do relativistic calculations correctly!

\section{The Antic Adjective}

My normal method of note-taking (write it all down and sort it out later), invariably yields a certain number of thoughts on the borderline between science and sociology. Here are a few of the items from S240. 
Harmanec in his introduction pointed out that the regimes of double stars and spectroscopic binaries overlap considerably and increasingly, a point reinforced in a number of the other talks. Might this eventually lead to a merger of the commissions? This was suggested more than a decade ago and opposed by both commissions, especially the vice presidents, one of whom would necessarily have been forced out of the succession. Since the two commissions collaborated successfully in the present organization, the issue is clearly not urgent.

Aristotle, remarked Richards, was the first tomographer, deducing the spherical shape of the earth from its invariably circular shadow on the moon. Perhaps one should say the first scientific tomographer, since I am reasonably certain that our remote Zinjanthropan ancestors, seeing the shadow of a large, fierce animal in the jungle, did not require threedimensional information before running like hell.

No one now living has split Procyon with the naked eye, reported Mason, meaning with the eye as a detector, we deduced, not "without a telescope" (the unaided eye). In either case, it suggests organization of a star party, perhaps at Lick, where Schaeberle did it first with the $36^{\prime \prime}$ to remedy this defect.

Not too many time assignment committees give speckle astronomers time to observe binaries on 8-10 meter telescopes, noted Balega. And a critical look at the frequency with which binary star papers are cited compared to other hotter topics (Trimble \& Zaich 2005) does not lead one to think this is likely to change in the foreseeable future.

The HR diagram really stands for Hans Rosenberg, claimed speaker Valls-Gabaud. And indeed he (a student of Karl Schwarzschild just before the latter moved to Potsdam) did make the first plot of apparent brightness vs. spectral type, for stars in the Pleiades, shortly before Hertzsprung and Russell had the same sort of idea (Rosenberg 1910). I have not attempted to find out what happened to Rosenberg over the next 30-plus years.

"You are lucky if you can find even a weak hydrogen line" in a white dwarf spectrum, according to Oswalt. The majority of white dwarfs are type DA, and the lines are not, in an equivalent-width sense, at all weak. They are, however, very wide, so that perhaps wavelength resolution beyond the $200 \AA / \mathrm{mm}$ of the hundreds of prime focus 200 -in plates I measured long ago is not an improvement.

The entries in the Washington Double Star Catalogue are known to be bound (concordant parallaxes or common proper motion) in about $2 \%$ of the cases, unbound (discordant proper motions or distances) in another $2 \%$ and undetermined for the rest, said Mason. Clearly one could repeat something like Michell's analysis (but using a better description of the distribution over the sky of stars of various apparent magnitudes) and decide which of those $2 \%$ 's is the tip of the iceberg. I cannot begin to guess whether this would take an afternoon or a decade for someone skilled in the use of globes.

The description of image restoration methods by ten Brummelaar has to be seen to be fully appreciated, but, in outline, speckles flash past your eyes; adaptive optics is a form of forcible flattening; and interferometry tears off a tiny bit of the information and discards the rest. The radio version, according to P.A.G. Scheuer, was like being led blindfolded up the side of a mountain by a single path, and then being asked to describe not only the entire mountain but its Fourier transform.

"It's worth rereading for anyone who hasn't read it before," was the description of the Popper (1980) article on stellar masses provided by Henry. Don't miss it if you can, in other words. Popper came from a family that had been in Northern California for a number of generations, but they were Jewish, and the presence of the name (an unlikely one somehow) on the walls of one of the Prague synagogues suggests that they may have come earlier from this part of the world. 
"Like so many of us, he fell in love with astronomy as a little boy," was McAlister's description of his undergraduate mentor, who had attended the IV ${ }^{\text {th }}$ General Assembly in Cambridge in 1932. This was the earliest GA to have had a daily newspaper as far as I have been able to determine. It was edited by Cecilia Payne (later Payne Gaposchkin), who had fallen in love with science when...

\section{Last Thoughts}

Several participants toward the end of the discussions expressed the view that the program had been a bit disconnected, perhaps a predictable result of the merging of two proposals and the concerns of both C26 and C42, and one might invoke the view of Santa Barbara cartoonist Ashleigh Brilliant that "the only requirement for getting there is to keep going in the right direction." This presupposes that one is sure which is the right direction (there being no one of whom one can ask directions in this context). The wide range of problems discussed and the high probability of some interesting binary products arising in more than one way more nearly recalls the claim of Peter De Vries that every novel should have a beginning, a muddle, and an end, and Carl Hansen's gloss that "we are now in the stellar muddling stage" (Hansen et al. 2004). If so, then the correct approach is that of Lord Ronald in the short story "Gertrude the Governess," who, according to author Stephen Leacock, "jumped on his horse and rode madly off in all directions.

It is the traditional prerogative of the last speaker at a meeting to offer thanks to the hosts and organizers, which I did and do, most heartily, and to say the last, slightly sad, word, in the form of farewell until the next conference, au revoir, auf weidersehn, do vidzenia, arrividerci, hasta la vista, tot ziens, or, in the language of our hosts, $\mathrm{Na}$ shledanou. In the end, however, Colin Scarfe had the last word, to remind us that Leacock was a Canadian, not an English, author!

\section{References}

Benson, R.S. 1970, unpublished Ph.D. thesis, U. California, Berkeley

Gyldenkerne, K. \& West, R.M. (Eds.) 1970, Mass Loss and Evolution in Close Binaries. Copenhagen University Publications, p. 25

Hansen, C.J. et al. 2004, Stellar Interiors. Springer p. 329

Hockey, T. et al. 2007, Biographical Encyclopedia of Astronomers. Springer (in press)

Michell, J. 1767, Phil. Trans. Roy. Soc. 57, 234

Paczyński, B. 1971, ARA \& A 10, 183

Rosenberg, H. 1910, Astron. Nach. 186, 71

Trimble, V. \& Zaich, P. 2005, PASP 117, 111

van der Hucht, K. (ed.) 2007, Highlights of Astronomy. Vol. 14, Cambridge Univ. Press 